ing capacity for oxygen transfer at each step.

The maximum capacities of several steps could be determined for comparison with actual aerobic capacity. For example, the final step in the mammalian respiratory system is oxygen uptake by mitochondria. Experiments in vitro ${ }^{4}$ showed that $1 \mathrm{ml}$ of muscle mitochondria has a maximal oxygen consumption of about $6 \mathrm{ml} \mathrm{O}_{2} \mathrm{~min}^{-1}$ with physiological substrates such as succinate. From actual aerobic capacities and total mitochondrial volumes of each species, Weibel and colleagues calculated that in peak aerobic exercise virtually all skeletal muscle mitochondria of individuals of each species are operating at almost full capacity. That is, there is a close match between theoretical mitochondrial capacity and actual peak load, with little reserve capacity left. In contrast, for the oxygendiffusing capacity of the lung some reserve remains unused even during a sprint, ranging from 50 per cent of total capacity for a big languid steer to only 20 per cent for a small athletic dog. The heart's ability to deliver oxygen similarly exceeds aerobic capacity by a modest reserve $^{5,6}$.

Detailed physiological and morphometric studies of individual steps reveal how each step's capacity is adjusted interspecifically to accommodate the 1,000 -fold range in aerobic capacity among the mammalian species studied. The simplest case is for oxygen uptake by muscle mitochondria, because there is no evidence of species differences in individual mitochondria. Instead, species with greater aerobic capacities simply have proportionately greater total mitochondrial volumes (for instance 40 times greater in a big swift horse than in a small slow goat; remarkably, Weibel et al. actually devised sampling procedures for estimating total mitochondrial volume in these animals). All those mitochondria consume oxygen at a rate of up to $5 \mathrm{ml} \mathrm{O}_{2} \mathrm{~min}^{-1}$ for each millilitre of mitochondria.

The next simplest case is for oxygen transfer across muscle capillaries. All species end up with $1.8 \mathrm{ml}$ of erythrocytes in their muscle capillaries for each $\mathrm{ml} \mathrm{O}_{2} \mathrm{~min}^{-1}$ of aerobic capacity, but species with bigger aerobic capacities achieve higher capillary erythrocyte volume in two different ways - smaller species just by having a proportionately greater relative capillary volume, more athletic species partly in that way and partly by possessing a higher haematocrit (erythrocytes constitute a greater fraction of their blood volume).

So muscle mitochondria achieve greater capacity by increasing one factor alone, muscle capillaries by increasing two. Variation in three factors is involved in matching the oxygen content NATURE · VOL $355 \cdot 16$ JANUARY 1992

\section{Squeezing diamond from fullerenes}

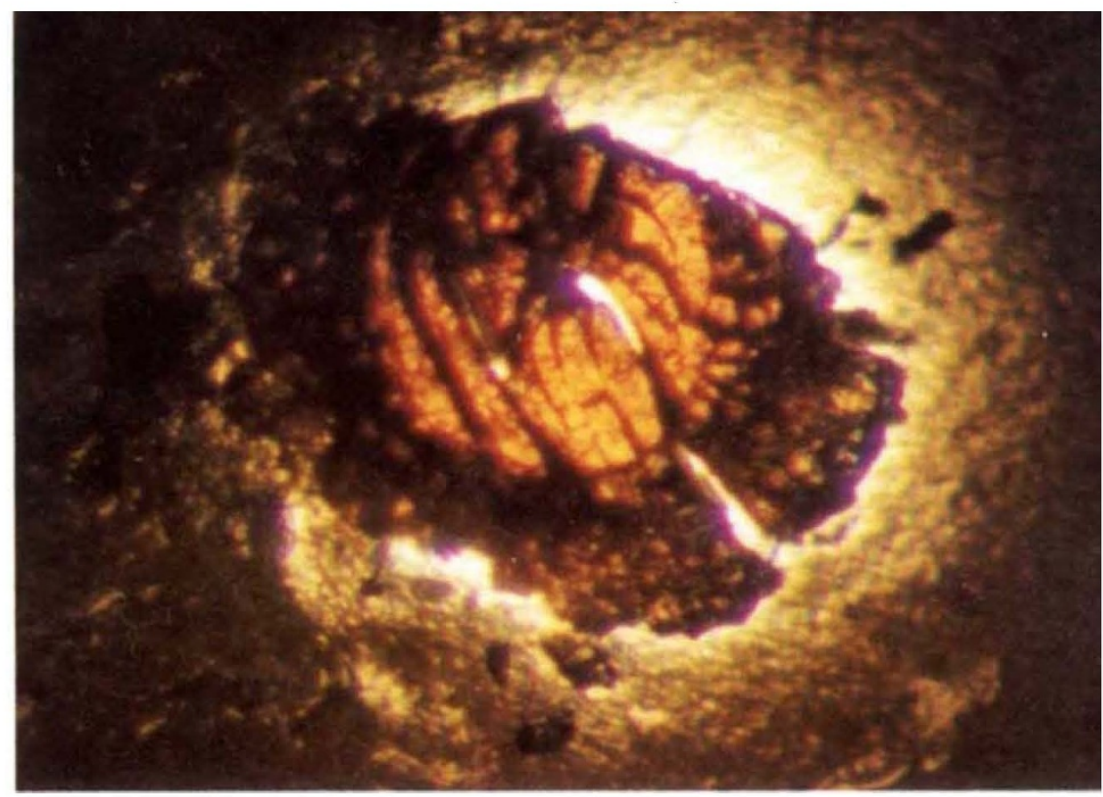

IT may not exactly look like a girl's best friend, but the diamond in the picture above has been formed as never before. Before being squeezed to pressures above 20 gigapascals (GPa) by Núñez Regueiro et $a l^{1}{ }^{1}$, the sample consisted of the black powder that is solid $\mathrm{C}_{60}$.

Interesting consequences have been predicted from compressing $\mathrm{C}_{60}$ crystals since they became available in macroscopic quantities. Perhaps they would become harder than diamonds ${ }^{2}$. Maybe they would link up to form an open, zeolite-type network ${ }^{3}$. Duclos et al. ${ }^{4}$ showed evidence for a change of phase around $16 \mathrm{GPa}$ under non-hydrostatic (anisotropic) compression. The possibility of a pressure-induced transition to a metallic phase motivated earlier experiments by Núñez Regueiro and colleagues $^{5}$; but instead they found a new, insulating phase at 15-20 GPa.

Little could be said then about the nature of this phase, although the authors assumed that theirs was the same as that of Duclos et al., which had been shown by X-ray diffraction to be of low symmetry, and that it contained an appreciable degree of $s p^{3}$ (saturated) bonding between carbon atoms. Were it not for the potential application of their

of blood pumped by the heart to aerobic capacity. Hearts of athletic species pump more oxygen per minute, partly because their blood has a higher haematocrit, partly because the heart itself is larger and pumps more blood per stroke. Hearts of larger species pump more oxygen per minute, partly because the heart is, of course, larger, but the observed relative constancy of heart size (about 0.58 per cent of body mass for large or small mammals) would leave small mammals undersupplied with oxy- results to diamond synthesis, the realization of Núñez Regueiro et al. that their present high-pressure phase ${ }^{1}$ is familiar old diamond might disappoint those hoping for an entirely new form of solid carbon. But there is no guarantee that the new phases seen in the earlier experiments $\mathrm{s}^{4.5}$ were diamond too.

The formation of diamond by squeezing is not unprecedented - graphite too can do the rearranging act, but less readily. Its phase boundary lies at a mere $1.6 \mathrm{GPa}$ at room temperature, but because of the very different types of bonding in the two materials, graphite will not make the transition even if compressed far beyond this. Only when shocked to $30-50 \mathrm{GPa}$ or heated above $1,200 \mathrm{~K}$ in the presence of a catalyst will graphite convert to diamond. But unlike graphite, $\mathrm{C}_{60}$ contains a degree of $s p^{3}$ as well as $s p^{2}$ (unsaturated) hybridization, fullerene molecules being nonplanar, and this presumably contributes to the ease of the structural transformation at room temperature ${ }^{1}$.

Phillp Ball

2. Ruoff, R. S. \& Ruoff, A. L. Nature 350, 663-664 (1991)

3. O'Keeffe, M. Nature 352. 674 (1991)

4. Duclos, S. J. et al. Nature 351, 380 (1991)

5. Núñez Regueiro, M. et al. Nature 354, 289 (1991).

gen, because the metabolic rate for each gram of tissue is greater for smaller animals. The necessary accommodation of small mammals depends entirely on their higher heartbeat frequency. Combining the effects of these three factors, the hearts of all mammals - regardless of size or athletic prowess - end up pumping about $2.8 \mathrm{ml}$ erythrocytes $\mathrm{min}^{-1}$ for each $\mathrm{ml} \mathrm{O}_{2} \mathrm{~min}^{-1}$ of aerobic capacity.

These examples make clear that tests of symmorphosis based on comparative
1. Núñez Regueiro, M. et al. Nature 355, 237 (1992). 